\title{
Do bulges of early- and late-type spirals have different morphology?
}

\author{
K. Fathi ${ }^{1,2}$ and R. F. Peletier ${ }^{1,2}$ \\ 1 School of Physics \& Astronomy, University of Nottingham, Nottingham, NG7 2RD, UK \\ ${ }^{2}$ CRAL, Observatoire de Lyon, 69561 St-Genis Laval Cedex, France
}

Received 7 October 2002 / Accepted 12 May 2003

\begin{abstract}
We study HST/NICMOS $H$-band images of bulges of two equal-sized samples of early- $\left(T_{\mathrm{RC} 3} \leq 3\right)$ and late-type spiral (mainly Sbc-Sc) galaxies matched in outer disk axis ratio. We find that bulges of late-type spirals are more elongated than their counterparts in early-type spirals. Using a KS-test we find that the two distributions are different at the $98.4 \%$ confidence level. We conclude that the two data sets are different, i.e. late-type galaxies have a broader ellipticity distribution and contain more elongated features in the inner regions. We discuss the possibility that these would correspond to bars at a later evolutionary stage, i.e. secularly evolved bars. Consequent implications are raised, and we discuss relevant questions regarding the formation and structure of bulges. Are bulges of early-type and late-type spirals different? Are their formation scenarios different? Can we talk about bulges in the same way for different types of galaxies?
\end{abstract}

Key words. galaxies: spiral - galaxies: bulges - galaxies: structure - galaxies: statistics

\section{Introduction}

Understanding bulges of galaxies is of pivotal importance for improving our overall theories for galaxy formation and evolution. Yet today, our general understanding of bulges is limited, and little is known about the formation scenario they have gone through. Up to the 1980 's, ideas about bulge formation were very much influenced by Baade's population concept (e.g. Sandage 1986). Since in our Galaxy the stars in both the bulge and the stellar halo were found to be old, it was thought that the bulges and the stellar galaxy halo were a single entity, with a common $r^{\frac{1}{4}}$ surface brightness (SB) law (de Vaucouleurs 1948, 1959). They were thought to be formed early on in the lifetime of the galaxy, during a rapid collapse phase (Eggen et al. 1962) before the formation of the disk. Their shapes were thought to be oblate spheroids (Kent et al. 1991). In recent years, however, a wealth of new data has altered our view of galactic bulges. Misalignments of the major axis with respect to the disk major axis indicate that bulges are probably not oblate (Bertola et al. 1991). Not all stars in the bulge region are old (e.g. Böker et al. 2002). And the surface brightness profiles of many bulges do not follow the $r^{\frac{1}{4}}$ law - there are claims nowadays that there are no bulges for which the surface brightness profiles obey this law (Balcells et al. 2003). Bulges do seem to be rotationally flattened (Kormendy \& Illingworth 1982; Davies \& Illingworth 1983), with some bulges rotating even faster, almost as fast as disks (Kormendy 1993).

Send offprint requests to: $\mathrm{K}$. Fathi, e-mail: ppxkf@nottingham.ac.uk
Before delving into the subject, it is good to first clearly define what we mean by bulge. In this paper, by bulge we mean the photometric inner component of the galaxy which appears to be superimposed onto an exponential disk. This definition is extremely simple, but is at the same time very robust. The definition is purely photometric, and is parametrical, but nonparametric studies by e.g. Kent (1986) show that the bulge obtained in this way is generally rounder than the disk, an indication that this definition is probably measuring something physical. Note that the definition does not depend on kinematics. When one measures the photometry of bulges defined in this way one finds that surface brightness profiles for galaxies of type earlier than Sc rise more steeply than those of latetypes. Surface brightness profiles generally are well described by the Sérsic (1968) $r^{\frac{1}{n}}$ profile, for which the value of the shape parameter $n$ varies from values around 4 for bulges of earlytype spirals, to 1 for bulges of late-type spirals (Andredakis et al. 1995; Phillips et al. 1996; Moriondo et al. 1998). Bulges also host a variety of central components including dust lanes, star forming rings and spiral structure reaching all the way to the centre (Zaritsky et al. 1993; Peletier et al. 1999; Carollo et al. 2002). Many bulges also contain central resolved sources, identified as star clusters (Carollo et al. 2002; Böker et al. 2001), which are fainter and less abundant in early-type spiral galaxies. Studying the nuclear properties of bulges is important, since they generally scale with the global galaxy properties (Faber et al. 1997). By studying the nuclear properties of bulges of early and late-type spirals, one might be able to provide the relative importance of dynamical effects in forming 
and/or maintaining the nuclear structure. It also provides a crucial test for formation scenarios of spheroidal stellar systems along the entire luminosity sequence.

The main theories about bulge formation include scenarios such as: primordial collapse where bulge and disk form sequentially (Eggen et al. 1962); hierarchical galaxy formation (Kauffmann \& White 1993); galaxy mergers or infall of satellite galaxies (Zinn 1985; Aguerri et al. 2001); and secular evolution (Kormendy 1979; Norman et al. 1996; Hasan et al. 1998), where central bars, formed by disk instabilities, thicken gradually to form bulges. The theories are still of crude, and theoretical predictions up to now have not been good enough for observers to rigorously test these scenarios. Effects of flattening by rotation, or thickening of the bars have not directly been verified in simulations for large numbers of objects, mainly due to lack of fast computers. There are a number of crude predictions that one would naively draw from these formation models. If bulges were formed by secular evolution of bars, they would in general be flatter than if they were made in a primordial collapse. One might object against such simplified predictions, but since there is no alternative until robust results from simulations are available, we will assume that these predictions are correct.

In this paper, we investigate the ellipticity distribution of a large sample of early- and late-type spirals, to ultimately be able to infer information about the formation scenario of bulges. Given that in the infrared, the effects of dust extinction is considerably less than in optical, HST/NICMOS $H$-band data are ideal for this task. We discuss a sub-sample of late-type spirals consisting of HST-archival data, together with a control sample of early-type spirals consisting of HST-archival images of both Seyferts and non-Seyferts from Laine et al. (2002) (hereafter L02). Since the NICMOS field of view is not large enough to provide Bulge-Disk decompositions, we use WFPC2 optical images for determination of the bulge radius, the radius where the light of the disk starts dominating the galaxy light. Our samples are large enough to allow us to draw statistical conclusions using the KS-test. The outline of the paper is as follows: In Sect. 2 we introduce our data sets, and describe the reduction procedure. In Sect. 3 the analysis procedure is outlined, and Sect. 4 includes an extensive discussion of our results and corresponding implications.

\section{The data}

We study a sample of archival HST/NICMOS (NIC2) F160W ( $\lambda_{\mathrm{e}} \approx 1.6 \mu \mathrm{m}$, roughly corresponding to $H$-band) data. The instrument and its performance are described in Thompson et al. (1998) and the NICMOS instrument handbook (MacKenty et al. 1997). For collecting the data the well known large observational data sets including samples by Mulchaey $I D=7330$, Peletier $I D=7450$, Pogge $I D=7867$, and Stiavelli $I D=$ 7331, were searched and investigated. These four samples are the only large samples of bulges in the HST archive, and include galaxies covering a wide range of morphological types. Of these samples, all galaxies of type later than or equal to $T_{\mathrm{RC} 3}=4$ (corresponding to $\mathrm{Sbc}$ ) are studied, and the earlier types are selected from the L02 sample. The * cal.FITS images were used as a starting point, after which additional data reduction steps were performed. Processed by the CALNICA pipeline (NICMOS Data Handbook), these images have been science calibrated, dark subtracted, flat fielded, and cosmic ray corrected in an automatic way. Visual inspection of the images showed a number of anomalies, and it was evident that some further reduction needed to be implemented.

Sky subtraction was done by subtracting the mean value of 10 randomly chosen outer regions of each image. Since in this study we are mainly interested in the central morphology, it is not important that the galaxies are much larger than the NIC2 frame. Masking bad pixels was done in two steps. First, only the most evident regions were masked by fitting planes interpolated from the boundary values (using our own software). This was followed by fitting ellipses to the isophotes (using STSDAS/ELLIPSE), and investigating the residual images, which showed the presence of additional undesired regions, such as very bright star forming knots, foreground stars, or very strong dust regions. After further masking of these regions, all the obvious artifacts were removed, and the images were ready for final ellipse fitting. The ELLIPSE task in the ISOPHOTE package in STSDAS was used on the masked images, this time providing more accurate results. The isophotes of each galaxy were fitted with a sequence of ellipses with different semi-major axis lengths based on the algorithm described by Jedrzejewski (1987). The final ellipses were then deprojected, using a twodimensional deprojection procedure assuming that the galaxy was a thin disk, with inclination (given by the axis ratio in the outer parts) and PA inferred from the RC3 catalogue (similar to the method used in L02). The deprojected fits are hereafter used in our analysis. Although removing the bad areas, before fitting ellipses, is believed to not seriously affect the fitting procedure (Carollo et al. 1998), we can be sure that the statistics are not affected by masking bad regions, since both our samples are treated in the same manner.

\subsection{Sample selection}

We decided to split the sample of bulges at $T_{\mathrm{RC} 3}=4$, since our previous studies have shown that bulges of spirals with types earlier than that have several properties in common with ellipticals, such as surface brightness (SB) profile, age, age spread, and agreement with the fundamental plane of ellipticals (e.g. de Jong 1996; Peletier et al. 1999; Falcón-Barroso et al. 2002). Bulges of late-type spirals have been shown to be different. We require that the galaxies are not too inclined, as this would imply large projection effects, and hide important features. Absolute $B$ magnitude, and distance (or $v_{\text {hel }}$ ) requirements are imposed, in order to avoid large differences in spatial resolution, and/or discrepant prominence of small scale structures such as stellar clusters or dust lanes. Small scale features are more prominent for nearby galaxies, whereas for more distant galaxies, we would not be able to see much of the inner parts. The same criteria were used when selecting the earlytype sample from L02, for which in addition we also rejected all the Seyfert 1 galaxies to avoid contamination of the central regions by the bright nucleus. 
Table 1. List of 35 rejected late-type galaxies and reasons for rejection (see text for further details). The remaining 35 galaxies fulfill all our criteria and match well with the early-type sample of L02.

\begin{tabular}{lcl}
\hline \hline Galaxy & $T_{\text {RC3 }}$ & Reason for rejection \\
\hline ESO 290-26 & 4 & Ellipse fitting fails (faint) \\
ESO 443-80 & 9 & Bulge never dominates \\
ESO 499-37 & 7 & Ellipse fitting fails (faint) \\
ESO 549-18 & 5 & Ellipse fitting fails (faint) \\
ESO 549-2 & 9 & Ellipse fitting fails (faint) \\
ESO 572-22 & 7 & Bulge never dominates \\
IC 1555 & 7 & Ellipse fitting fails (patchy) \\
NGC 151 & 4 & No B/D decomposition \\
NGC 406 & 5 & Bulge never dominates \\
NGC 578 & 5 & No B/D decomposition \\
NGC 1483 & 4 & Bulge never dominates \\
NGC 1800 & 9 & Ellipse fitting fails (patchy) \\
NGC 1892 & 6 & Bulge never dominates \\
NGC 2104 & 9 & Bulge never dominates \\
NGC 2336 & 4 & Matching with L02 sample \\
NGC 2748 & 4 & Bulge never dominates \\
NGC 2964 & 4 & Matching with L02 sample \\
NGC 3079 & 5 & Bulge never dominates \\
NGC 3259 & 4 & Matching with L02 sample \\
NGC 4536 & 4 & Matching with L02 sample \\
NGC 5054 & 4 & Matching with L02 sample \\
\hline
\end{tabular}

An initial sample of all bulges included 70 objects (all latetype), on which we applied further selection. Using parameters from the RC3 catalogue (de Vaucouleurs et al. 1991), we removed all the galaxies more inclined than $\simeq 63^{\circ}$ corresponding to the apparent outer, $R_{25}$ disk axis ratio of $b / a \leq 0.45$. Subsequently, the sample was restricted to a limited radial velocity range of $500 \mathrm{~km} \mathrm{~s}^{-1} \leq v_{\text {hel }} \leq 4000 \mathrm{~km} \mathrm{~s}^{-1}$, and absolute $B$-magnitude of $M_{B} \leq-15$ (all inferred from the $\mathrm{RC} 3$ catalogue). As we needed to estimate the bulge radius by performing a bulge-disk decomposition (see Sect. 2.2) on the corresponding WFPC2 images, we required availability of WFPC2 optical images on which ellipse fitting was possible. This rules out off-field galaxies (i.e. galaxies for which the central areas were only partly on the field), low SB galaxies and those with very strong star forming regions and/or strong dust features. Although the $H$-band images are believed to be unaffected by these effects, a few galaxies had to be removed for this purpose. Table 1 quantifies the number of rejected galaxies after application of each selection criterion. Finally, to obtain reliable statistics, it is of vital importance that the two samples are similarly distributed in all salient parameters. We compare our sample with the sample of L02 to make a matching control sample of early type spiral bulges, applying the same ranges in $M_{B}$, distance, and $i$, and since not many galaxies with $0.4<b / a<0.6$ are available in the sample of L02 we removed 5 galaxies from our late-type sample. Given that the very bright nucleus of type 1 Seyfert galaxies affects the central parts of the images, the Seyferts selected from the L02 sample are all of type 2 . The final remaining list of the two subsamples are found in Tables 2 and 3, and the corresponding distributions are illustrated in Fig. 1.

\subsection{Bulge-disk decomposition}

A very important quantity for our study is the "bulge radius". This is defined as the radius at which the SB of the exponential disk equals the surface brightness of the bulge. Successful fits to the bulges of spiral galaxies have been obtained using the Hubble law (Hubble 1930), King model (King 1966), de Vaucouleurs $r^{\frac{1}{4}}$ law (de Vaucouleurs 1948), by a generalised version of de Vaucouleurs' law $r^{\frac{1}{n}}$ (Caon et al. 1993; Andredakis et al. 1995) and by an exponential function (Kent et al. 1991; Andredakis \& Sanders 1994; Baggett et al. 1998). Andredakis \& Sanders (1994) and Andredakis et al. (1995) showed that for the late-type spirals of this paper an exponential fit of the bulge is entirely adequate, and much better than using an $r^{\frac{1}{4}}$ law.

We decomposed the galaxy SB profiles, fitting exponentials to both the bulge and the disk part. Despite the fact that potentially better fits can be obtained with a Sérsic $r^{\frac{1}{n}}$ law for the bulge, we decided to use exponential distributions, since these produce the most robust determinations for the bulge radius. As a test we have compared bulge radii obtained using a Sérsic + exponential distribution with bulge radii obtained with double-exponential distributions and found that both values are not very different in log-scale (see Table 4). We find that the measured bulge ellipticities do not change significantly when Sérsic bulge radii are used and our statistical conclusions are not affected. Since the field of view of NICMOS is too small to be able to perform the Bulge-Disk decomposition on, we used optical WFPC2 images for this purpose. The optically obtained bulge radii do not differ much from those from the $H$-band images (Carollo et al. 2002). Images with the F555W, F547M, F606W, and F814W filters were used with the galaxy nucleus generally on the Planetary Camera chip. A manual reduction (bad pixel masking, sky subtraction etc.) as described previously was performed on the mosaiced images, followed by ellipse fitting. As a result of the high sensitivity of the optical images to dust extinction, the axis ratio of the successive ellipses was fixed (axis ratio and PA as used for the deprojection) throughout the galaxy. The resulting average radial SB profile is then decomposed into bulge and disk, and the bulge radius is obtained.

\section{Analysis}

The reduced data are now ready to be analysed. In Fig. 7 we plot the deprojected ellipticities and PAs, obtained from the ellipse fitting, as a function of radius. We used the bulge radii, from the bulge-disk decomposition, to estimate the extent of the bulge region. In this region we quantified the bulge ellipticities in the following way: When the ellipticity profile within the bulge varies by less than 0.1 , we define the bulge ellipticity as the mean value. Alternatively, when there is a rise or fall 
Table 2. List of 35 remaining late-type galaxies. The first four columns indicate galaxy name, morphological Type, outer disk axis ratio $b / a$, and radial velocities from the RC3 catalogue. The three ellipticity columns are the derived projected, deprojected, and average bulge ellipticities as described in the text, and the derived $B / T$ ratio using Eq. (1). The last column is the bulge radius obtained from bulge-disk decomposition of the WFPC2 images. The $\dagger$ marks galaxies for which a nuclear bar has been detected, i.e. we have found an ellipticity variation greater than 0.1 within the bulge region.

\begin{tabular}{|c|c|c|c|c|c|c|c|c|}
\hline Galaxy & Type & $b / a$ & $c z\left(\mathrm{~km} \mathrm{~s}^{-1}\right)$ & $\epsilon_{\mathrm{cenPR}}$ & $\epsilon_{\mathrm{cenDEP}}$ & $\epsilon_{\text {average }}$ & $B / T$ & $r_{B}(\operatorname{arcsec})$ \\
\hline ESO 404-G3 & .SBT4P. & 0.50 & 2383 & 0.65 & 0.40 & 0.35 & 0.013 & 0.80 \\
\hline ESO 498-G5 & .SXS4P. & 0.81 & 2413 & 0.10 & 0.15 & 0.15 & 0.024 & 1.86 \\
\hline IC 5273 & .SBT6* & 0.66 & 1206 & 0.72 & 0.65 & 0.50 & 0.011 & 1.74 \\
\hline NGC 289 & .SBT4.. & 0.71 & 1690 & 0.30 & 0.10 & 0.15 & 0.009 & 1.14 \\
\hline NGC 1300 & .SBT4.. & 0.66 & 1592 & 0.10 & 0.30 & 0.30 & 0.030 & 2.29 \\
\hline NGC $1345^{\dagger}$ & .SBS5P* & 0.74 & 1543 & 0.70 & 0.40 & 0.45 & 0.394 & 9.15 \\
\hline NGC $1688^{\dagger}$ & .SBT7.. & 0.78 & 1223 & 0.60 & 0.60 & 0.50 & 0.323 & 0.88 \\
\hline NGC 1961 & .SXT5.. & 0.65 & 3983 & 0.25 & 0.40 & 0.35 & 0.009 & 1.86 \\
\hline NGC 2276 & .SXT5.. & 0.96 & 2372 & 0.25 & 0.25 & 0.20 & 0.005 & 0.78 \\
\hline NGC $2339^{\dagger}$ & .SXT4.. & 0.76 & 2361 & 0.35 & 0.45 & 0.30 & 0.025 & 2.15 \\
\hline NGC 2344 & .SAT5* & 0.98 & 914 & 0.05 & 0.05 & 0.05 & 0.045 & 1.89 \\
\hline NGC 2903 &. SXT4.. & 0.48 & 565 & 0.22 & 0.40 & 0.40 & 0.003 & 1.18 \\
\hline NGC $3145^{\dagger}$ & .SBT4.. & 0.51 & 3656 & 0.35 & 0.55 & 0.40 & 0.062 & 2.54 \\
\hline NGC 3949 & .SAS4* & 0.58 & 681 & 0.35 & 0.25 & 0.25 & 0.012 & 0.75 \\
\hline $\mathrm{NGC} 4030^{\dagger}$ & .SAS4.. & 0.72 & 1449 & 0.15 & 0.30 & 0.25 & 0.025 & 2.59 \\
\hline NGC $4303^{\dagger}$ & .SXT4.. & 0.89 & 1607 & 0.30 & 0.35 & 0.15 & 0.002 & 2.44 \\
\hline NGC $4806^{\dagger}$ & .SBS5?. & 0.83 & 2430 & 0.20 & 0.25 & 0.25 & 0.004 & 0.91 \\
\hline NGC 4939 & .SAS4.. & 0.51 & 3091 & 0.35 & 0.25 & 0.25 & 0.037 & 2.72 \\
\hline NGC 5005 & .SXT4.. & 0.48 & 992 & 0.55 & 0.50 & 0.20 & 0.078 & 1.95 \\
\hline NGC $5033^{\dagger}$ & .SAS5.. & 0.47 & 861 & 0.45 & 0.40 & 0.30 & 0.035 & 1.63 \\
\hline NGC 5427 & .SAS5P. & 0.85 & 2645 & 0.05 & 0.15 & 0.10 & 0.017 & 1.61 \\
\hline NGC 5643 & .SXT5.. & 0.87 & 1163 & 0.10 & 0.15 & 0.10 & 0.052 & 1.07 \\
\hline NGC $6000^{\dagger}$ & .SBS4* & 0.87 & 2110 & 0.55 & 0.55 & 0.25 & 0.055 & 1.65 \\
\hline NGC $6217^{\dagger}$ & RSBT4.. & 0.83 & 1368 & 0.35 & 0.45 & 0.25 & 0.141 & 2.56 \\
\hline NGC $6221^{\dagger}$ & .SBS5.. & 0.69 & 1350 & 0.35 & 0.40 & 0.20 & 0.027 & 2.40 \\
\hline NGC 6384 & .SXR4.. & 0.66 & 1690 & 0.30 & 0.15 & 0.15 & 0.079 & 2.37 \\
\hline NGC $6412^{\dagger}$ & .SAS5.. & 0.87 & 1475 & 0.34 & 0.45 & 0.20 & 0.006 & 1.87 \\
\hline NGC 6744 & $. S X R 4 .$. & 0.65 & 730 & 0.20 & 0.25 & 0.25 & 0.016 & 1.70 \\
\hline NGC 6814 & .SXT4.. & 0.93 & 1509 & 0.05 & 0.10 & 0.10 & 0.054 & 1.98 \\
\hline NGC 6951 & .SXT4.. & 0.83 & 1331 & 0.19 & 0.15 & 0.15 & 0.030 & 2.13 \\
\hline NGC 7126 & .SAT5.. & 0.46 & 2980 & 0.30 & 0.40 & 0.40 & 0.057 & 2.24 \\
\hline NGC 7188 & PSBT4.. & 0.47 & 1767 & 0.15 & 0.45 & 0.40 & 0.009 & 0.57 \\
\hline NGC 7392 & .SAS4.. & 0.59 & 2908 & 0.34 & 0.25 & 0.25 & 0.018 & 2.13 \\
\hline NGC 7421 & .SBT4.. & 0.89 & 1830 & 0.20 & 0.20 & 0.20 & 0.027 & 1.44 \\
\hline NGC 7479 & .SBS5.. & 0.76 & 2394 & 0.35 & 0.20 & 0.20 & 0.023 & 1.12 \\
\hline
\end{tabular}

in the ellipticity of more than 0.1 , we take the peak ellipticity. These two definitions provide the bulge ellipticity for the whole of our sample (see the histograms in bins of 0.1 in Fig. 2). As we are comparing the results with that of a control sample, any conclusion investigating whether the two datasets are statistically significant needs to be based on a statistical test of the two samples.
We used the Kolmogorov-Smirnov KS-test, which has the advantage of making no initial assumption about the distribution of data, i.e. it is non-parametric and distribution free (Press et al. 1992). For our deprojected ellipticity distributions we obtain a KS probability value of 0.0160 , meaning that the two distributions are different at the $98.4 \%$ confidence level. 
Table 3. List of 35 remaining early-type galaxies. The columns are the same as in Table 2. Error values for the ellipticities are of order $<0.002$. For the $B / T$ ratio, the errors are of order $<0.005$, and for $r_{B}$ the errors are of order $<0.5 \operatorname{arcsec}$. (Note that the errors for Table 2 are of same order.)

\begin{tabular}{|c|c|c|c|c|c|c|c|c|}
\hline Galaxy & Type & $b / a$ & $c z\left(\mathrm{~km} \mathrm{~s}^{-1}\right)$ & $\epsilon_{\text {cenPR }}$ & $\epsilon_{\mathrm{cenDEP}}$ & $\epsilon_{\text {average }}$ & $B / T$ & $r_{B}(\operatorname{arcsec})$ \\
\hline ESO 137 G-34 & .SXS0?. & 0.76 & 2620 & 0.15 & 0.15 & 0.15 & 0.013 & 3.80 \\
\hline IC $2560^{\dagger}$ & PSBR3*. & 0.63 & 2873 & 0.25 & 0.35 & 0.15 & 0.020 & 1.77 \\
\hline NGC 1365 & .SBS3.. & 0.55 & 1675 & 0.20 & 0.55 & 0.40 & 0.008 & 1.10 \\
\hline NGC $1530^{\dagger}$ & .SBT3.. & 0.52 & 2506 & 0.20 & 0.60 & 0.40 & 0.019 & 3.13 \\
\hline NGC $1672^{\dagger}$ & $. S B S 3 .$. & 0.83 & 1282 & 0.15 & 0.20 & 0.10 & 0.110 & 1.76 \\
\hline NGC 2460 & .SAS1.. & 0.76 & 1442 & 0.25 & 0.20 & 0.20 & 0.027 & 2.13 \\
\hline NGC 2639 & RSAR $1 * \$$ & 0.60 & 3198 & 0.20 & 0.20 & 0.20 & 0.032 & 2.22 \\
\hline NGC 3032 &. $\mathrm{LXR0..}$ & 0.89 & 1568 & 0.10 & 0.05 & 0.05 & 0.037 & 1.76 \\
\hline NGC 3081 & RSXR0.. & 0.78 & 2391 & 0.20 & 0.30 & 0.30 & 0.027 & 2.38 \\
\hline NGC $3169^{\dagger}$ & .SAS1P. & 0.63 & 1261 & 0.25 & 0.25 & 0.20 & 0.020 & 1.95 \\
\hline NGC 3227 & SXS1P. & 0.68 & 1145 & 0.20 & 0.20 & 0.20 & 0.028 & 1.65 \\
\hline NGC 3277 & .SAR2.. & 0.89 & 1460 & 0.10 & 0.05 & 0.05 & 0.470 & 0.56 \\
\hline NGC 3300 &. $\mathrm{LXR} 0 * \$$ & 0.52 & 3045 & 0.30 & 0.35 & 0.35 & 0.025 & 2.35 \\
\hline NGC 3982 & .SXR3* & 0.87 & 924 & 0.20 & 0.10 & 0.10 & 0.015 & 3.58 \\
\hline NGC 4117 &. L... $0^{*}$ & 0.49 & 871 & 0.30 & 0.25 & 0.25 & 0.008 & 2.21 \\
\hline NGC 4143 & .LXSO.. & 0.63 & 784 & 0.20 & 0.20 & 0.20 & 0.105 & 2.13 \\
\hline NGC $4151^{\dagger}$ & PSXT2* & 0.71 & 956 & 0.20 & 0.20 & 0.20 & 0.068 & 1.53 \\
\hline NGC 4260 & .SBS1.. & 0.50 & 1886 & 0.30 & 0.30 & 0.30 & 0.008 & 2.36 \\
\hline NGC 4384 & .S...1.. & 0.78 & 2400 & 0.60 & 0.60 & 0.60 & 0.084 & 4.18 \\
\hline NGC 4725 & .SXR2P. & 0.71 & 1180 & 0.10 & 0.25 & 0.25 & 0.047 & 2.12 \\
\hline NGC $4941^{\dagger}$ & RSXR2*. & 0.54 & 846 & 0.40 & 0.30 & 0.25 & 0.009 & 2.21 \\
\hline NGC 5064 & PSA. $2^{*}$ & 0.46 & 2952 & 0.50 & 0.20 & 0.20 & 0.046 & 2.36 \\
\hline NGC 5273 & .LAS0.. & 0.91 & 1054 & 0.15 & 0.05 & 0.05 & 0.020 & 3.04 \\
\hline NGC 5377 & RSBS1.. & 0.56 & 1830 & 0.30 & 0.30 & 0.30 & 0.026 & 2.13 \\
\hline NGC $5383^{\dagger}$ & PSBT $3 * \mathrm{P}$ & 0.85 & 2226 & 0.25 & 0.20 & 0.10 & 0.014 & 2.58 \\
\hline NGC $5448^{\dagger}$ & RSXR1.. & 0.46 & 1973 & 0.40 & 0.20 & 0.20 & 0.023 & 2.13 \\
\hline NGC 5614 & .SAR2P. & 0.83 & 3872 & 0.05 & 0.20 & 0.20 & 0.078 & 1.60 \\
\hline NGC $5678^{\dagger}$ & .SXT3.. & 0.49 & 2267 & 0.30 & 0.25 & 0.25 & 0.015 & 1.77 \\
\hline NGC 5953 &.$S A .1 * P$ & 0.83 & 2099 & 0.20 & 0.25 & 0.25 & 0.127 & 2.17 \\
\hline NGC $6300^{\dagger}$ & .SBT3.. & 0.66 & 1064 & 0.30 & 0.30 & 0.20 & 0.006 & 2.93 \\
\hline NGC 7496 & .SBS3.. & 0.91 & 1527 & 0.40 & 0.35 & 0.20 & 0.016 & 2.45 \\
\hline NGC 7217 & RSAR2.. & 0.83 & 935 & 0.05 & 0.10 & 0.10 & 0.026 & 2.58 \\
\hline NGC 7716 & .SXR3* & 0.83 & 2541 & 0.30 & 0.25 & 0.15 & 0.028 & 3.79 \\
\hline NGC $7742^{\dagger}$ & .SAR3.. & 1.00 & 1661 & 0.10 & 0.10 & 0.05 & 0.031 & 1.61 \\
\hline NGC 7743 & RLBS+.. & 0.85 & 1722 & 0.10 & 0.15 & 0.15 & 0.019 & 2.61 \\
\hline
\end{tabular}

To find out whether this result is real we applied a number of tests. We started by comparing the projected ellipticities of early and late type spirals. The corresponding distributions of the projected bulge ellipticities are given in Fig. 3. The two distributions are peaked at different values (e.g. there is a significant excess of late-type galaxies with bulge ellipticities in the $0.3-0.4$ bin, and a deficit for the $0.2-0.3 \mathrm{bin}$ ). The bulges of late-type spirals are more flattened even when comparing the projected ellipticities, although in this case the KS-test leads to a probability value of 0.0630 , corresponding to $93.7 \%$ confidence level. The projected ellipticities are consistent with a single distribution, not necessarily implying the same after deprojection. 

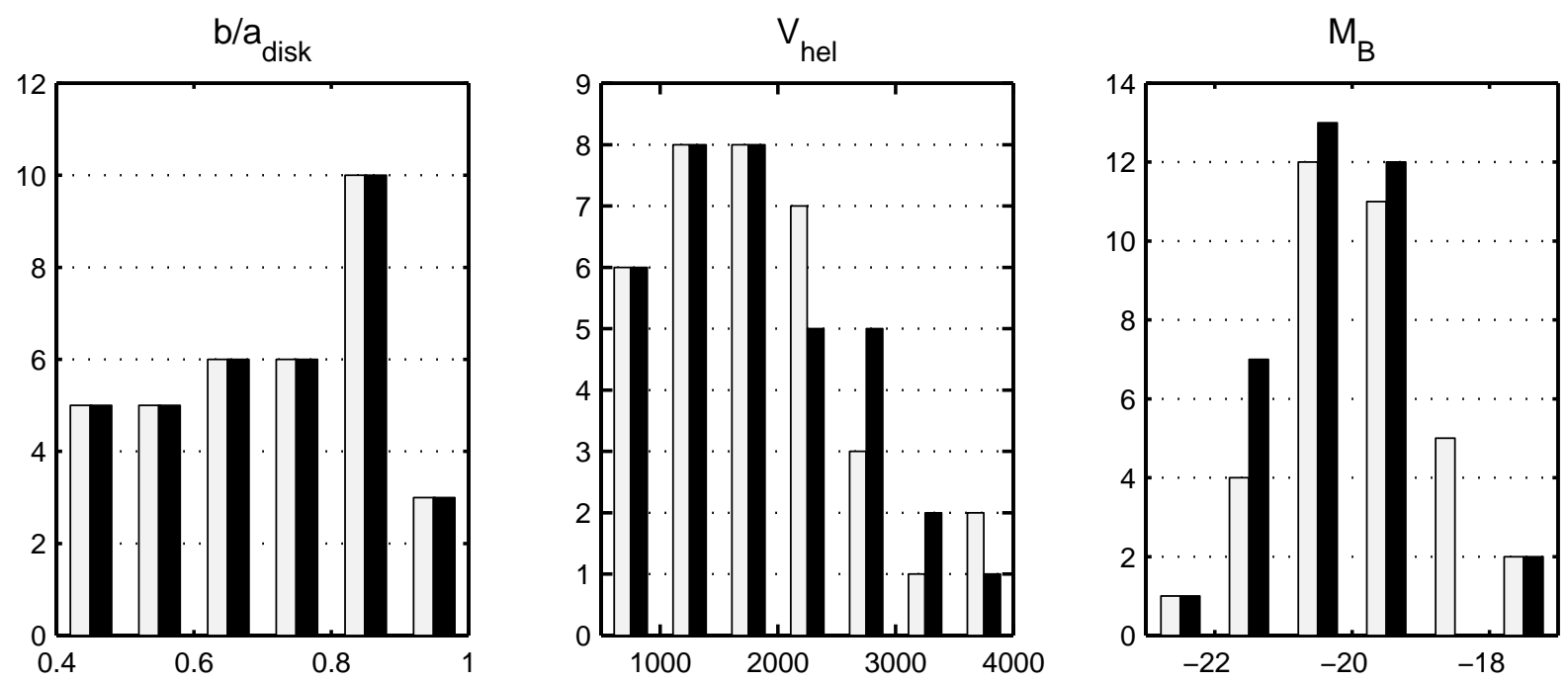

Fig. 1. Distribution histograms of $b / a, v_{\text {hel }}$, and $M_{B}$ of the two samples, The black colour represents the early-type control sample and the lighter colour represents the late-type sample. Each subsample contains 35 galaxies, all of which fulfil all the selection criteria described in the text above, and for obtaining the $M_{B}, H_{0}=70 \mathrm{~km} \mathrm{~s}^{-1} \mathrm{Mpc}^{-1}$ was used.

Table 4. Average bulge radii (in arcsec) with corresponding rms (in brackets) obtained from fitting Sérsic + exponential and doubleexponential SB profiles to the WFPC2 images.

\begin{tabular}{lcc}
\hline \hline & $\overline{r_{B}}[$ Sér $+\operatorname{Exp}]$ & $\overline{r_{B}}[\operatorname{Exp}+\operatorname{Exp}]$ \\
\hline Early-types & $6.04(3.27)$ & $2.30(0.76)$ \\
Late-types & $3.80(2.16)$ & $1.95(1.40)$ \\
\hline
\end{tabular}

We continue by defining the bulge ellipticity in a different way, namely such that in all the cases we would take this value to be the average ellipticity of the bulge component of the galaxy. This provides a different distribution (see Fig. 4), with a KS-test result of 0.3200 . This implies that the ellipticity distribution of bulges in early and late type spirals is similar, and that the only difference between the two groups is the structure inside the bulge.

Investigating the residual images after fitting ellipses (Fig. 8) shows that in 12 cases the residual images are very smooth all the way to the very centre of the galaxy. This means that the galaxy light is very well described by the set of fitted ellipses This fact shows that the galaxy does not contain very much dust, and that the ellipticity analysis is a very reliable indicator of the distribution of the light. In the remaining 23 cases, the residual images show strong central features meaning that there are significant structures in the central parts. These are disks, bars, strong star formation regions, spiral structure, and/or dust features. In these cases the residual images also show clear indications of dusty bulges for which the inferred morphology is affected by the dust (e.g. the position of the centre changes as a function of radius).

\section{Discussion}

\subsection{Possible observational bias}

We have found that the distribution of bulge ellipticities is different between early and late-type spirals (see Fig. 2). The KS-test indicates however that the statistical significance of this result is small. Could it be that we made unjustified assumptions during the deprojection? We discuss here a number of observational biases which could cause this result and find out whether they might be significant.

The difference between the ellipticity distributions might be caused by the inferred 2D deprojection. This procedure assumes that the galaxy is two dimensional, which in reality is not the case. Depending on the thickness variations along the galaxy plane the "deprojected" bulge may seem more elongated as an artifact of the 2D deprojection. If the bulge is much thicker than the underlying disk it becomes more elongated, and consequently if bulges of late-type galaxies are intrinsically thicker than those of early-types, our statistics could be biased. For this purpose we analysed the intrinsic bulge thickness of a sample of 20 edge-on galaxies in the $K$-band and in the same Hubble-type range as our main sample (Guijarro Roman et al., in preparation). The sample consists of 14 early-type spiral galaxies and 6 late-type spirals, a subset of the sample of de Grijs (1998). For the 20 galaxies we derived the bulge scale heights by directly fitting exponentials to the vertical light profile, and the bulge scale length through $B / D$ decomposition of the radial profile (as described in Sect. 2.2). The ratio of the bulge scale height $\left(h_{z}\right)$ to the bulge scale length $\left(h_{r}\right)$ directly measures the bulge axis ratio, and a comparison of these value tells whether the bulge of one galaxy is flatter or thicker than another. Figure 5 shows that this ratio is not dependent on type, and that bulges of early-type spirals are not intrinsically thicker than bulges of late-type spirals, or vice versa. Hence, the 2D deprojection does not add any bias to our statistics for comparing the deprojected ellipticities. 

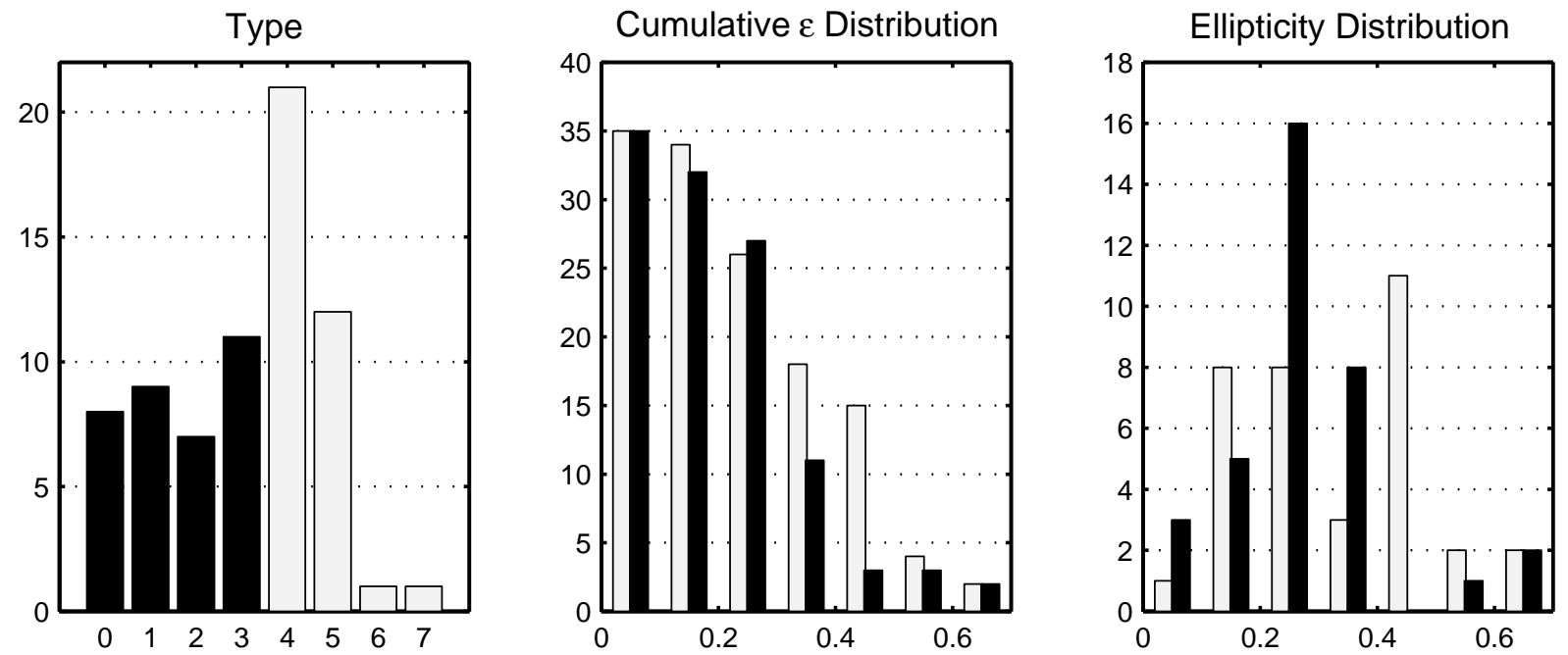

Fig. 2. Histograms of type distribution, deprojected bulge ellipticity ( $\left.\epsilon \equiv 1-\frac{b}{a}\right)$ cumulative distribution, and bulge ellipticity distribution (in order from left to right) of our late-type and early-type samples. The black colour represents the early-type subsample.



Fig. 3. The distribution of projected bulge ellipticities for our earlyand late-type samples. The black colour represents the early-type sample.

It could be that the difference is caused by the fact that it is more difficult to detect elongated structures in big bulges of early-type spirals. We can investigate that by looking at the frequency of bars detected inside the bulge regions. Defining bars in the same way as in Knapen et al. (2000) we find no difference in the frequency of bars in the bulge region between early- and late-type galaxies. In our sample, 12 early-type and 13 late-type spirals indicate the presence of a bar within the bulge. These numbers are very similar, and no discrepancy is observed.

Instead of galaxy type we might also look at the bulge to total $(B / T)$ flux ratio, given by

$\frac{B}{T}=\frac{I_{0 B} \times h_{B}^{2}}{I_{0 B} \times h_{B}^{2}+I_{0 D} \times h_{D}^{2}}$,

where $I_{0 B}$ and $h_{B}$ denote the central intensity and the scale length of the bulge, respectively, and the index $D$ indicates the same parameters for the disk component.

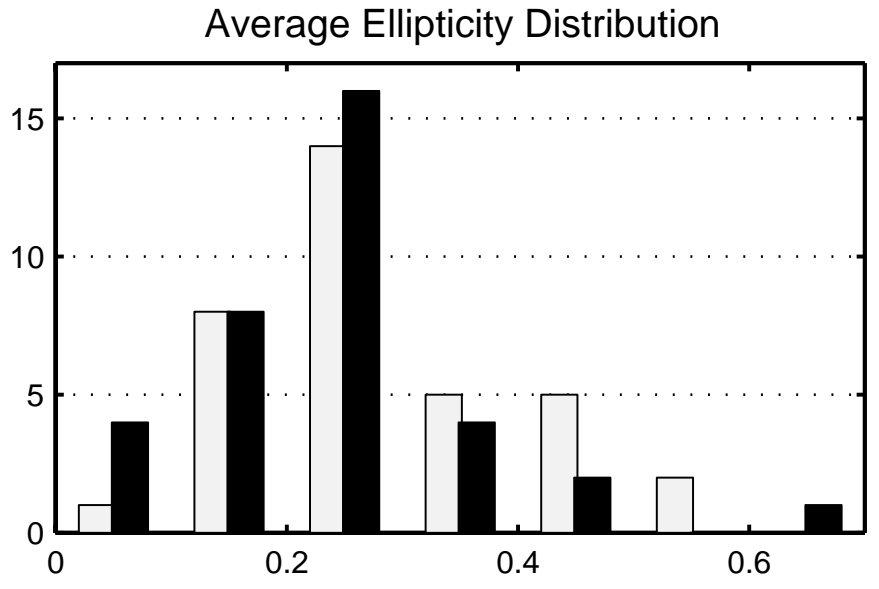

Fig. 4. Bulge ellipticity distribution for the average $\epsilon$ values. Colour convention is same as before.

We derive this parameter for our entire sample and divide the sample in two equal-sized subsamples according to this value, drawing the middle line. This results in two subsamples, one containing galaxies with $B / T$ ratio $<0.026$, and one containing the rest of the sample. The bulge peak ellipticities are then compared (see Fig. 6), and the corresponding KS-test probability value is 0.1975 . As also seen from the histogram, one cannot say that the distributions are different. Although one might think that the $B / T$ ratio is a good indicator of galaxy type, it is not a very good parameter for our sample here, since most $B / T$ ratios are very small (less than 0.05 ), and in this range of $B / T$ it is not a very good morphological type indicator.

\subsection{Implications}

We find that bulges of late-type galaxies are more elongated. What does this mean for the formation of bulges? Are we seeing some signs of secular evolution here? Could it be that secular evolution destroys nuclear bars, converting them slowly 




Fig. 5. $\frac{h_{z}}{h_{r}}$ vs. $h_{z}$ for 15 of the sample edge on galaxies. Filled black circles are the values for the early-type spirals. It is evident that intrinsic thickness is independent of galaxy type. Note also that 5 of our initial sample edge ons did not show to have a prominent bulge, and therefore were not taken into consideration, as the obtained $\frac{h_{z}}{h_{r}}$ vs. $h_{z}$ would be unreliable.

\section{$\varepsilon$ Distribution}

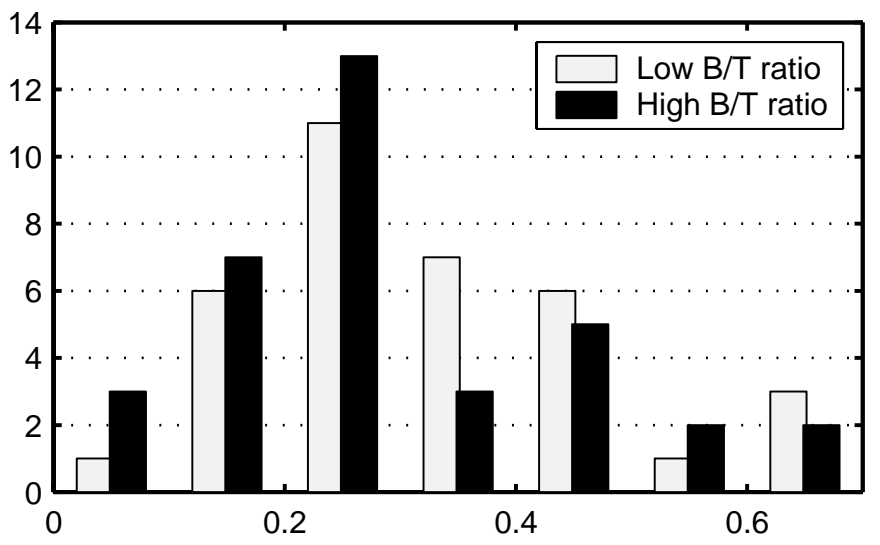

Fig. 6. Distribution of deprojected ellipticities as a function of $B / T$ ratio. The median $B / T$ ratio is $B / T=0.026$.

into bulges, which as a result are still somewhat more elongated?

Theoretical studies have shown that bars are capable of evolving in self dissolving mechanisms (Sellwood \& Wilkinson 1993), i.e. they undergo secular evolution (Toomre 1966; Hasan \& Norman 1990; Pfenniger \& Norman 1990). Secular evolution, however, is not always believed to destroy the bars. Merritt \& Sellwood (1994) showed that the thickening of bars, through off plane bending instabilities, stops when the density in the mid-plane drops to a low enough value that the natural vertical frequency for a large fraction of particles drops below the forcing frequency below the global bend. There is also some strong theoretical evidence that long lived and strong bars survive loss of up to $2 / 3$ of the angular momentum and reduced pattern speed by a factor of up to 5 (Sellwood \& Debattista 1996).

Spiral activity has been shown to occur for a longer time in the outer disk when the initial bar ends at a radius well inside the outer edge of the disk. This spiral activity in the disk outside the bar is not affected by the bar, although the bar grows by trapping additional particles which are ready to lose angular momentum near the inner ends. The new particles added to the bar in this way still have too much angular momentum to sink deep into the bar, and are therefore added to the outer ends of the bar. As a result, the angular momentum of the bar content is rising although the bar itself is slowing down. Bar destruction and pattern speed change are also explained with other theories. Interaction with the halo (Weinberg 1985; Debattista \& Sellwood 1996; Athanassoula 1996) is believed to be able to slow down a bar through dynamical friction. Mergers (Gerin et al. 1990; Athanassoula 2002; Heller \& Shlosman 1994; Barnes \& Hernquist 1998), or fuelling of an active nucleus by driving gas towards the centre, play a considerable role in destruction of bars resulting in a spheroidal bulge (Norman et al. 1996).

It is not obvious that secular evolution of bars leads to oblate structures. Secularly evolved bars may also end up as elongated structures, as bars are believed to be populated by orbits of greater eccentricity than those in the axisymmetric parts of the disk. One might think that bar formation, on the other hand, is expected to be less common in early-type systems (Kormendy 1979, 1993; Combes \& Elmegreen 1993; Sellwood \& Wilkinson 1993; Lütticke et al. 2000), as there is less gas in early-type spirals, and there is also already a big bulge which stabilises the galaxy and prevents self-gravitating bar formation. If secular evolution is active one would therefore expect to find more elongated structures in late-type spirals, as we find observationally. This argument, however, might not be correct, since recent statistics on bars (e.g. Lütticke et al. 2000) shows that the bar fraction in early type spirals (Sa's) is the same as in Sc's. Also, Athanassoula (2002) has recently shown that massive bulges do not always suppress bar formation, when more accurate simulations with live-particles bulges/halos are done. In any case, in this paper we do not see a big difference between shapes of central features in early and late type spirals, so the process which makes elongated bulges in late-type spirals is not much more efficient than in early-type spirals.

To summarise, secular evolution seems to be able to make bulges (central disk concentrations) from bars, but it is not clear what their final shape is, and how efficient this process is. Yet so far it is not possible to confirm nor refute any of these theories, as very few observational studies have been done (Gerssen et al. 1999; Zimmer \& Rand 2002), and only improved observations will be able to tell whether secular evolution of bulges is indeed possible.

\section{Summary}

Studying a sample of 70 galaxies in the $H$-band, we find that inner features in bulges of late-type spirals are more elongated than those in early-type spirals, although the statistical 

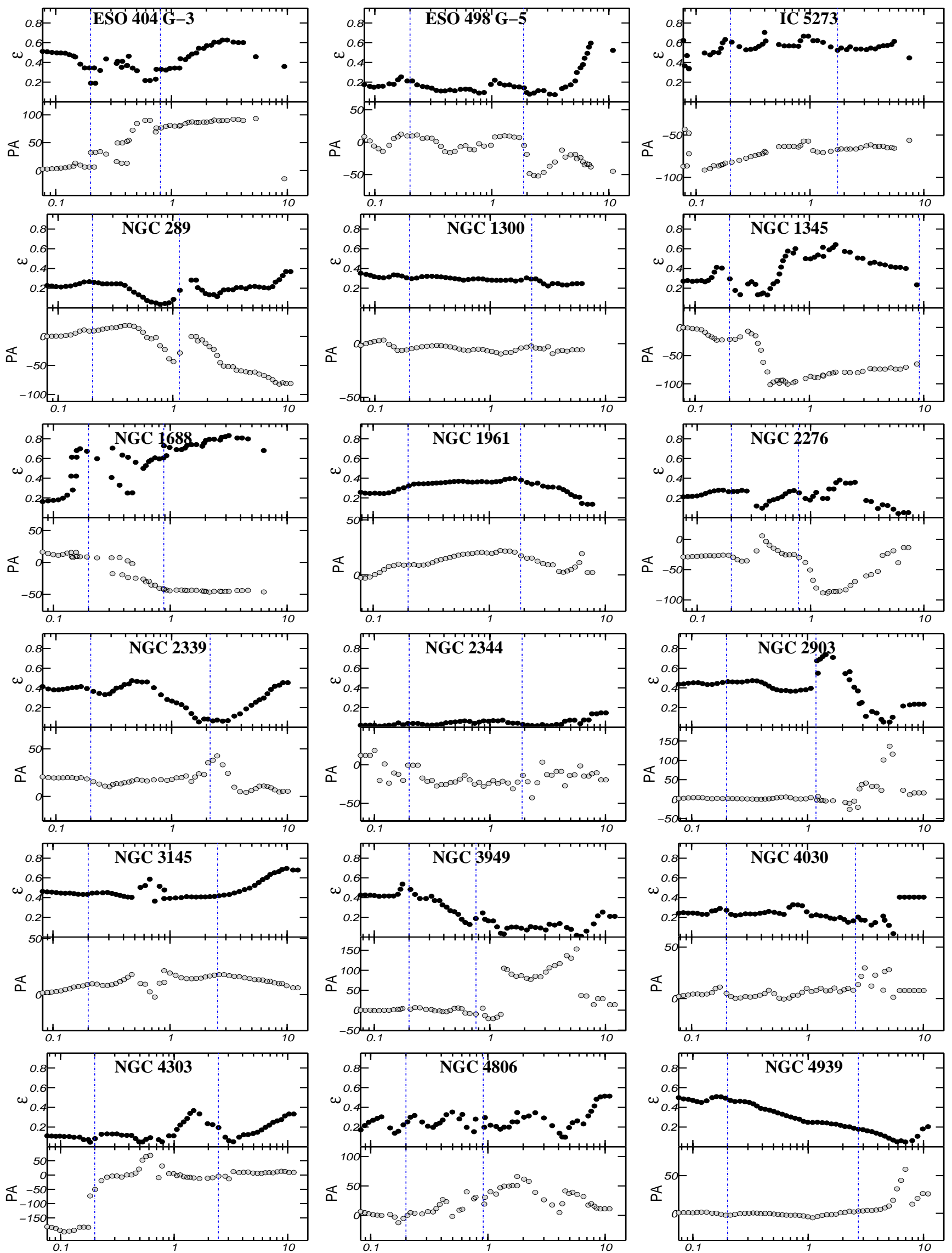

Fig. 7. Deprojected ellipticity (top) and PA (bottom) profiles for our late-type spirals. On the horizontal axis, the radius in arcseconds is given, and the dashed vertical lines indicate the inner and outer bulge radii. The inner bulge radius is defined as the region where ellipse fitting can be unreliable (e.g. Peletier et al. 1990; Rest et al. 2001) i.e. $\simeq 0.2^{\prime \prime}$. 

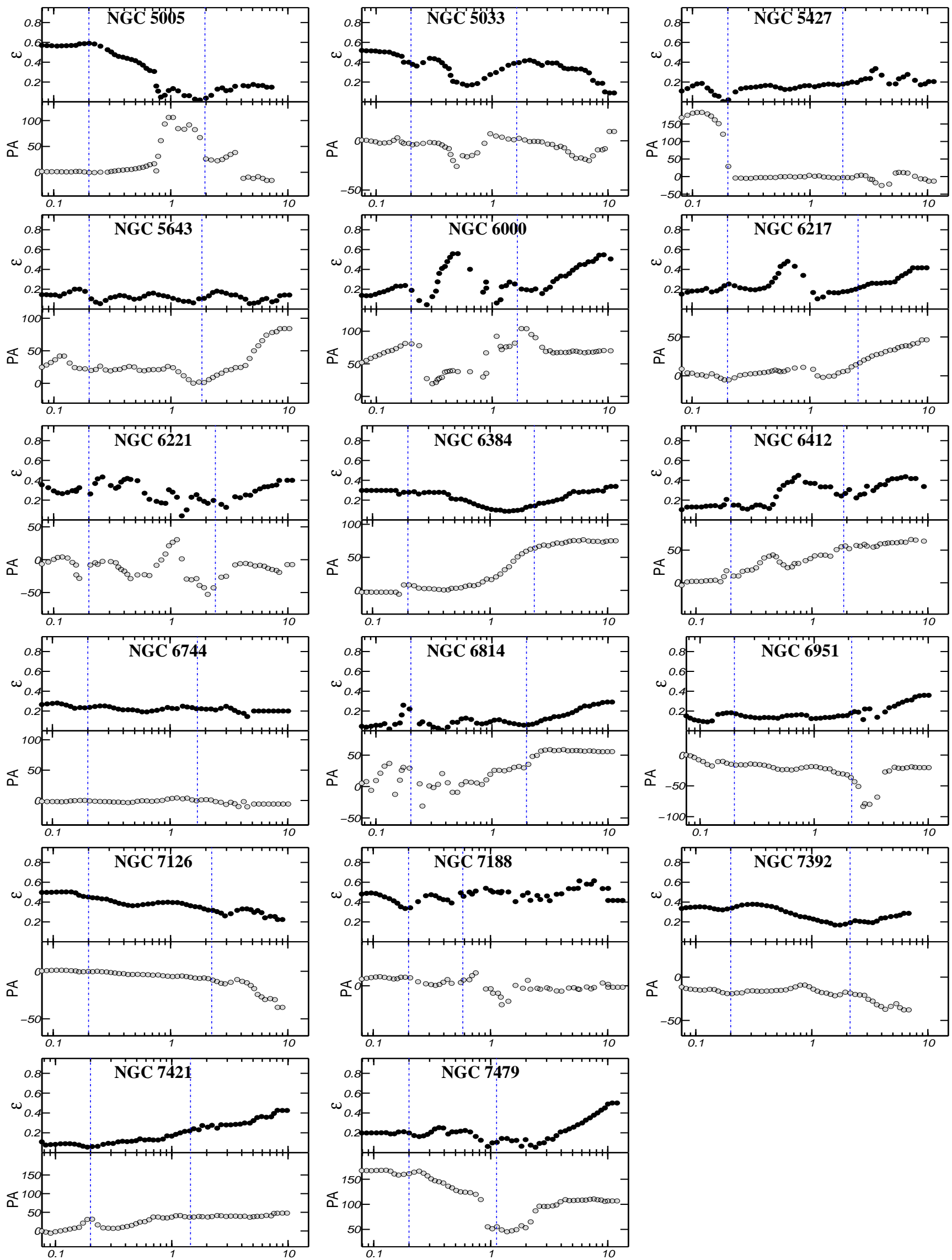

Fig. 7. continued. 

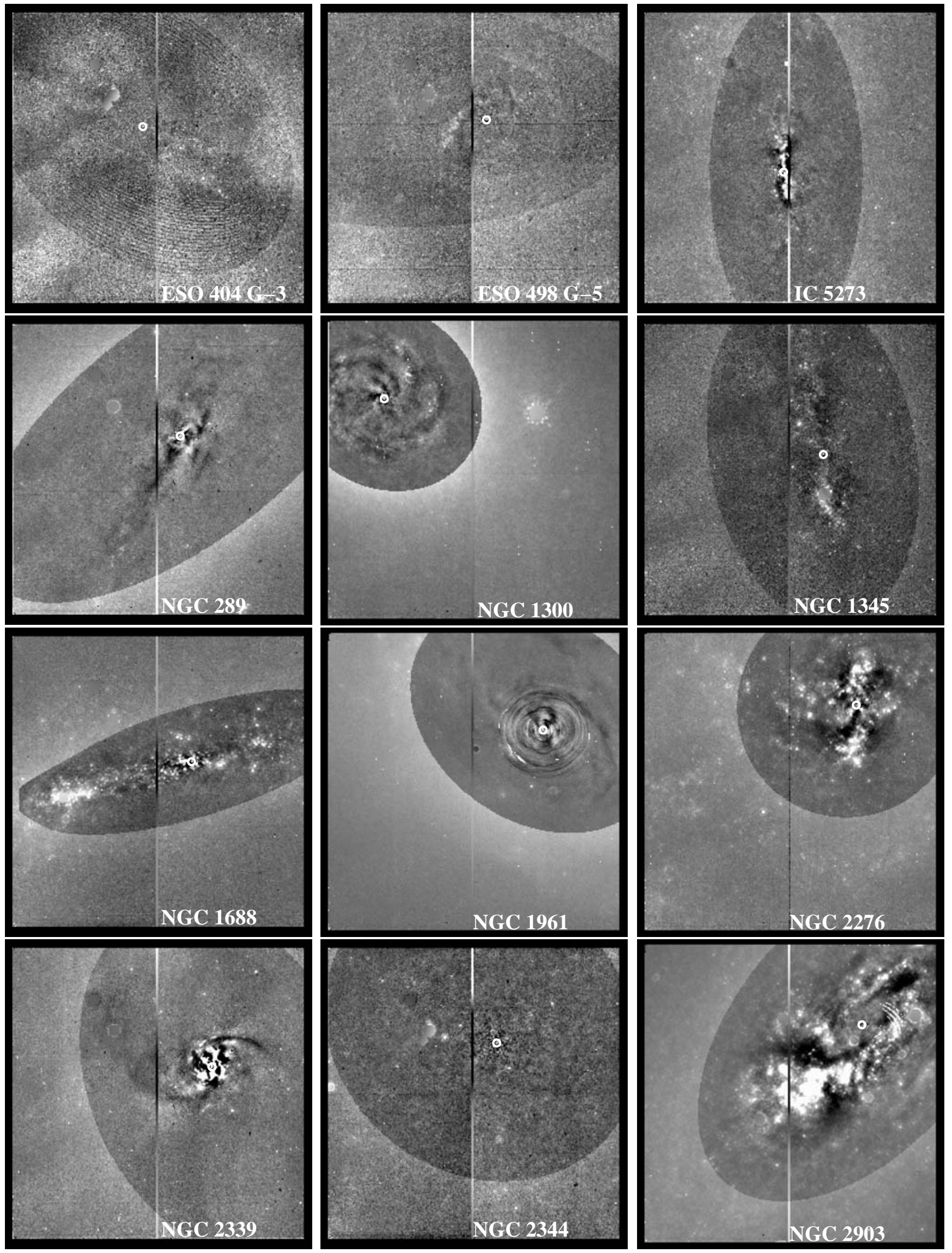

Fig. 8. HST/NICMOS (NIC2) $H$-band residual images (original image - model image generated from ellipse fitting). The field of view is $19^{\prime \prime} \times 19^{\prime \prime}$ and the pixel size is $0.075^{\prime \prime}$. The circular ring marks the estimated centre of the galaxy, and its size corresponds to the inner bulge radius. 

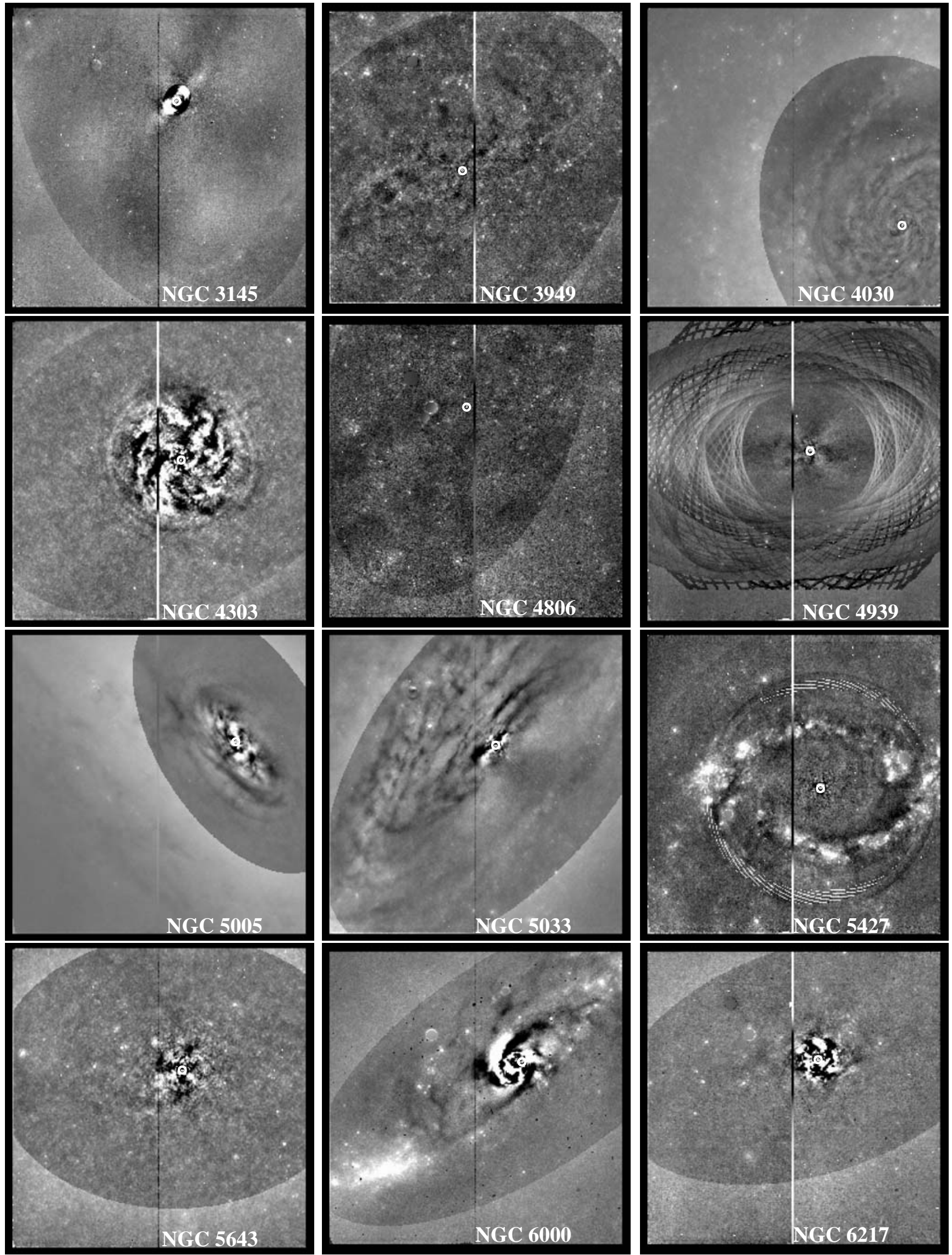

Fig. 8. continued. 

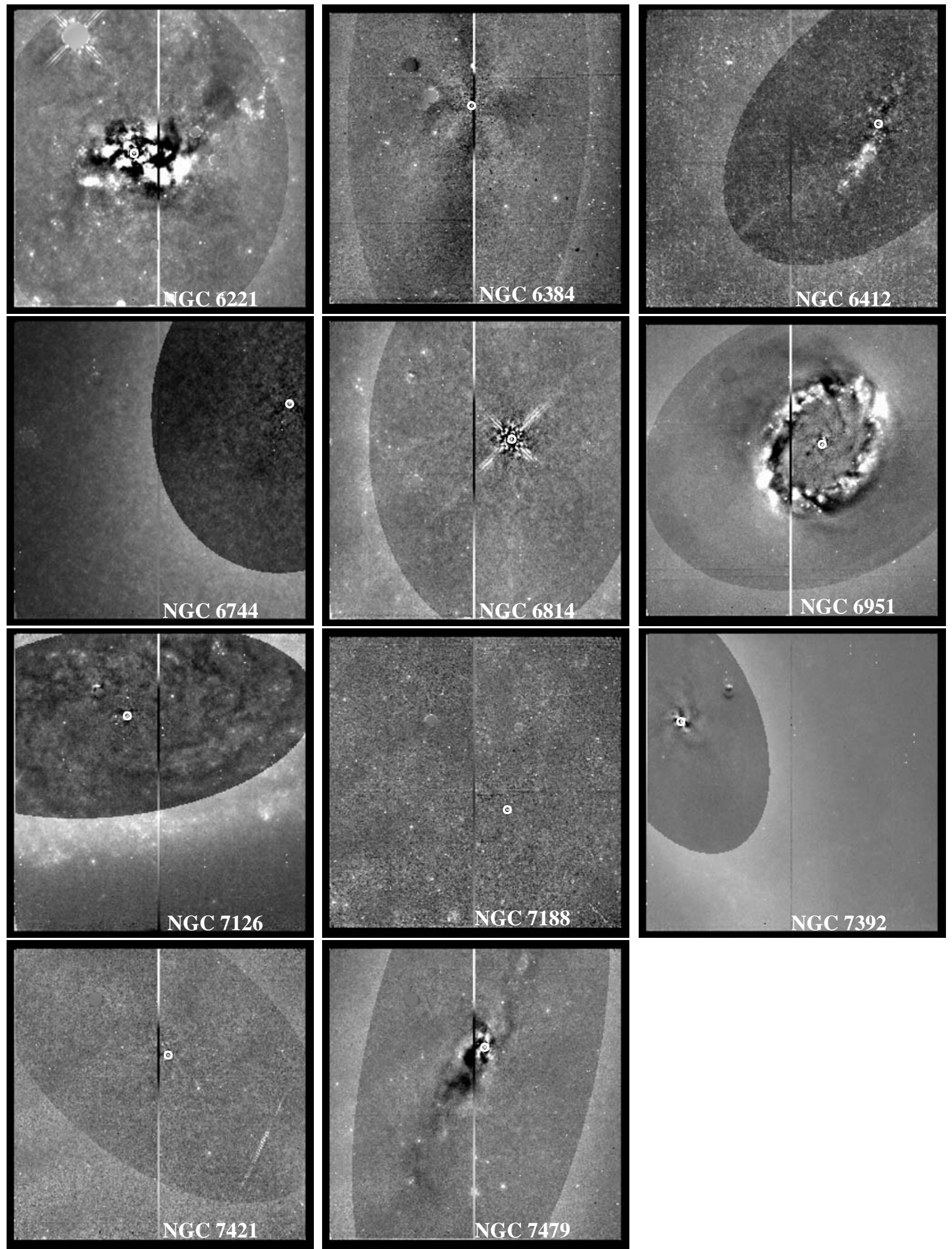

NGC 7392

Fig. 8. continued. 
significance of this result is small $\left(P_{\mathrm{KS}}=0.0160\right)$. When we simply compare the average ellipticity in the bulge between both samples, we don't find any difference. This probably indicates that bulges of later type spiral galaxies contain more elongated features like nucluear bars than bulges of early-type spirals. We have performed several tests to establish that this result is not due to observational effects: bars are visible just as easily in early-type bulges as in bulges of late type galaxies. Also, deprojection cannot cause the larger fraction of elongated features in late-type spirals.

The result could be explained if bulges of late-type spiral galaxies are formed primarily through secular evolution of bars, while this would not be the case for earlier-type bulges. Since however the morphology of bulges that have been created through secular evolution is still unclear, it is not possible at this stage to give more detailed conclusions. To better understand the formation process of bulges, it is important that we first obtain a better understanding of the process of secular evolution.

Acknowledgements. We thank Michael Merrifield for very interesting and useful discussions. The data presented in this paper were obtained from the Multimission Archive at the Space Telescope Science Institute (MAST). STScI is operated by the Association of Universities for Research in Astronomy, Inc., under NASA contract NAS5-26555. Support for MAST for non-HST data is provided by the NASA Office of Space Science via grant NAG5-7584 and by other grants and contracts. Much of the analysis was performed using IRAF which is distributed by NOAO.

\section{References}

Aguerri, J. A. L., Balcells, M., \& Peletier, R. F. 2001, A\&A, 367, 428 Andredakis, Y. C., \& Sanders, R. H. 1994, MNRAS, 267, 283

Andredakis, Y. C., Peletier, R. F., \& Balcells, M. 1995, MNRAS, 275, 874

Athanassoula, E. 1996, in Barred Galaxies, ed. R. Buta, D. A. Crocker, \& B. G. Elmegreen (San Francisco: ASP), IAU Colloq., 157, 309

Athanassoula, E. 2002, Ap\&SS, 281, 39

Baggett, W. E., Baggett, S. M., \& Anderson, K. S. J. 1998, AJ, 116, 1626

Balcells, M., Graham, A. W., Domínguez-Palmero, L., \& Peletier, R. F. 2003, ApJ, 582, L79

Barnes, J. E., \& Hernquist, L. 1998, ApJ, 495, 187

Bertola, F., Vietri, M., \& Zeilinger, W. W. 1991, ApJ, 374, L13

Böker, T., Laine, S., van der Marel, R. P., et al. 2002, AJ, 123, 1389

Böker, T., Laine, S., van der Marel, R. P., et al. 2001, AAS, 199, 0705

Caon, N., Capaccioli, M., \& D’Onofrio, M. 1993, MNRAS, 265, 1013

Carollo, C. M., Stiavelli, M., \& Mack, J. 1998, AJ, 116, 68

Carollo, C. M., Stiavelli, M., Seigar, M., de Zeeuw, P. T., \& Dejonghe, H. 2002, AJ, 123, 159

Combes, F., \& Elmegreen, B. G. 1993, A\&A, 271, 391

Davies, R. L., \& Illingworth, G. 1983, ApJ, 266, 516

Debattista, V. P., \& Sellwood, J. A. 1996, in Barred Galaxies, ed. R. Buta, D. A. Crocker, \& B. G. Elmegreen (San Francisco: ASP), IAU Colloq., 157, 357

de Grijs, R. 1998, MNRAS, 299, 595 de Jong, R. S. 1996, A\&A, 313, 45

de Vaucouleurs, G. 1948, AnAp, 11, 247

de Vaucouleurs, G. 1959, Hdb. d. Physik, 53, 311

de Vaucouleurs, G., \& Pence, W. D. 1978, AJ, 83, 1163

de Vaucouleurs, G., de Vaucouleurs, A., Corwin, J. R., et al. 1991, Third Reference Catalogue of Bright Galaxies (New York: Springer)

Eggen, O. J., Lynden-Bell, D., \& Sandage, A. R. 1962, ApJ, 136, 748

Faber, S. M., Tremaine, S., Ajhar, E. A., et al. 1997, AJ, 114, 1771

Falcón-Barroso, J., Peletier, R. F., \& Balcells, M. 2002, MNRAS, 335, 741

Gerin, M., Combes, F., \& Athanassoula, E. 1990, A\&A, 230, 37

Gerssen, J., Kuijken, K., \& Merrifield, M. R. 1999, MNRAS, 306, 926

Hasan, H., \& Norman, C. A. 1990, ApJ, 361, 69

Hasan, H., Pfenniger, D., \& Norman, C. A. 1998, AAS, 19310509

Heller, C. H., \& Shlosman, I. 1994, ApJ, 424, 84

Hubble, E. P. 1930, ApJ, 71, 231

Jedrzejewski, R. 1987, MNRAS, 226, 747

Kauffmann, G., \& White, S. D. M. 1993, MNRAS, 261, 921

Kent, S. M., Dame, T. M., \& Fazio, G. 1991, ApJ, 37813

Kent, S. M. 1986, AJ, 91, 1301

King, I. R. 1966, AJ, 71, 64

Knapen, J. H., Shlosman, I., \& Peletier, R. F. 2000, ApJ, 529, 93

Kormendy, J. 1979, ApJ, 227, 714

Kormendy, J., \& Illingworth, G. 1982, ApJ, 256, 460

Kormendy, J. 1993, IAUS, 153, 209

Laine, S., Shlosman, I., Knapen, J. H., \& Peletier, R. F. 2002, ApJ, 567, 97

Lütticke, R., Dettmar, R.-J., \& Pohlen, M. 2000, A\&AS, 145, 405

MacKenty, J. W., Tremaine, S., Ajhar, E. A., et al. 1997, NICMOS Instrument Handbook, version 2.0 (Baltimore: STScI)

Merritt, D., \& Sellwood, J. A. 1994, ApJ, 425, 551

Moriondo, G., Giovanardi, C., \& Hunt, L. K. 1998, A\&AS, 130, 81

Norman, C. A., Sellwood, J. A., \& Hasan, H. 1996, ApJ, 462, 114

Peletier, R. F., Davies, R. L., Illingworth, G. D., Davis, L. E., \& Cawson, M. 1990, AJ, 100, 1091

Peletier, R. F., Balcells, M., Davies, R. L., et al. 1999, MNRAS, 310 , 703

Phillips, A., Illingworth, G., Mackenty, J., \& Franx, M. 1996, AJ, 111, 1566

Pfenniger, D., \& Norman, C. 1990, ApJ, 363, 391

Press, H. W., Teukolsky, S. A., Vetterling, W. T., \& Flannery, B. P. 1992, Numerical recipes in FORTRAN. The art of scientific computing (Cambridge University Press)

Rest, A., van den Bosch, F. C., Jaffe, W., et al. 2001, AJ, 121, 2431

Sandage, A. 1986, ARA\&A, 24, 421

Sellwood, J. A., \& Wilkinson, A. 1993, Rep. Prog. Phys., 56, 173

Sellwood, J. A., \& Debattista, V. P. 1996, in Barred Galaxies and Circumnuclear Activity, ed. A. Sandqvist, \& P. O. Lindblad (Springer-Verlag), 43

Sérsic, J. L. 1968, Atlas de galaxias australes, Observatorio Astronomico Cordoba

Thompson, R. I., Rieke, M., Schneider, G., Hines, D. C., \& Corbin, M. R. 1998, ApJ, 492, L95

Toomre, A. 1966, in Geophysical Fluid Dynamics, 66-46, 111

Weinberg, M. D. 1985, MNRAS, 213, 451

Zaritsky, D., Rix, H.-W., \& Rieke, M. 1993, Nature, 364, 313

Zimmer, P., \& Rand, R. 2002, AAS, 200, 9701

Zinn, R. 1985, ApJ, 293, 424 